\title{
Factors predicting pathological upgrading after prostatectomy in patients with Gleason grade group 1 prostate cancer based on opinion-matched biopsy specimens
}

\author{
YUKI MARUYAMA $^{1}$, TAKUYA SADAHIRA ${ }^{1}$, MOTOO ARAKI $^{1}$, YOSUKE MITSUI $^{1}$, KOICHIRO WADA $^{1}$, \\ ACOSTA GONZALEZ HERIK RODRIGO ${ }^{1}$, KAZUAKI MUNETOMO ${ }^{2}$, YASUYUKI KOBAYASHI ${ }^{1}$, \\ MASAMI WATANABE ${ }^{1}$, HIROYUKI YANAI ${ }^{3}$, TOYOHIKO WATANABE ${ }^{1}$ and YASUTOMO NASU ${ }^{1}$ \\ Departments of ${ }^{1}$ Urology, ${ }^{2}$ Radiology and ${ }^{3}$ Pathology, Okayama University Graduate School of Medicine, \\ Dentistry and Pharmaceutical Sciences, Okayama 700-8558, Japan
}

Received June 24, 2019; Accepted December 11, 2019

DOI: $10.3892 / \operatorname{mco} .2020 .1996$

\begin{abstract}
The present study investigated the concordance between Gleason scores assigned to prostate biopsy specimens by outside pathologists and a urological pathology expert, and determined the risk of upgrading between opinion-matched Gleason grade group (GGG) 1 biopsy specimens and radical prostatectomy specimens. Between January 2012 and May 2018, 733 patients underwent robot-assisted radical prostatectomy. Patients whose original biopsy specimens from outside hospitals were reviewed by a urological pathology expert Okayama University Hospital were included. Patients who had received neoadjuvant hormonal therapy were excluded. Logistic regression analysis was used to identify predictors of upgrading among GGG 1 diagnoses. A total of 403 patients were included in the present study. Agreement in GGG between initial and second-opinion diagnoses was present in 256 cases (63.5\%). Although opinion-matched cases improved concordance between biopsy and prostatectomy specimen GGG compared with single-opinion cases (initial, $35.2 \%$; second-opinion, $36.5 \%$; matched, $41.4 \%$ ), $71 \%$ (56/79) of cases classified as GGG 1 were upgraded after prostatectomy. Multivariate analysis revealed that prostate-specific antigen density and Prostate Imaging Reporting and Data System version 2 score were significant predictors of upgrading (odds ratio, 1.10; $\mathrm{P}=0.01$; and odds ratio, $1.88 ; \mathrm{P}=0.03$, respectively). In conclusion, the GGG concordance rate between needle-core biopsy and radical prostatectomy specimens was higher in opinion-matched cases; however, $71 \%$ of opinion-matched
\end{abstract}

Correspondence to: Dr Takuya Sadahira, Department of Urology, Okayama University Graduate School of Medicine, Dentistry and Pharmaceutical Sciences, 2-5-1 Shikata-cho, Kita-ku, Okayama 700-8558, Japan

E-mail: t.sadahira@gmail.com

Key words: prostate cancer, biopsy, radical prostatectomy, second opinion, Gleason grade
GGG1 cases were upgraded after robot-assisted radical prostatectomy. Urologists should propose treatment strategies or further biopsy rather than active surveillance for patients with GGG1 and a high PSAD and/or PI-RADS score.

\section{Introduction}

The Gleason scoring system is an essential tool for predicting tumor aggressiveness and affects selection of a prostate cancer (PC) treatment strategy (1). However, the Gleason score (GS) often differs between needle-core biopsy (NCB) and radical prostatectomy (RP) specimens (2). Such discordance occurs in $28-76 \%$ of patients, and is associated with many factors, including the number and length of biopsy cores, and more significantly, pathologist misreading $(2,3)$. To reduce these inconsistencies, several groups have recommended the adoption of mandatory second-opinion review by specialized urologic pathologists when patients are referred from other clinics (4-6). Second opinions change the GS of some specimens, resulting in the alteration of therapeutic management strategies (5-7). As a mandatory review program at our institution, outside specimens are routinely reviewed by a specialized urologic pathologist before treatment.

Recently, a consensus conference revised PC pathological grading into five grade groups using a system that offers the highest prognostic discrimination $(1,8)$. This new grading system readjusted the grading scale by designating GS $3+3$ to grade 1, and more accurately reflects tumor behavior by differentiating between GS 3+4 (grade 2) and GS 4+3 (grade 3) and between GS 4+4, 3+5, and 5+3 (grade 4) and GS 4+4, 5+4, $5+5$ (grade 5). Of these prognostic groups, patients are most commonly assigned to Gleason grade group (GGG) 1, which is most sensitive to discordance with respect to treatment strategy (9). This occurs because more than $40 \%$ of men with low-risk prostate cancers (PCs) now receive active surveillance as initial treatment $(10,11)$. Therefore, upgrading of GGG exposes patients at an increased risk of disease progression.

Despite previous reports of upgrading predictors, the results were affected by pathologist misreading (11). This problem can be regarded as resolved when the initial and 
second pathologist opinions reach a concordant diagnosis. These studies compared the diagnostic accuracy between original and second opinions of RP specimens; however, the agreement between RP specimen and NCB specimen diagnoses by two pathologists has not been previously evaluated. In this study, we selected patients for whom a consensus diagnosis was achieved based on NCB specimens, and identified predictors of upgrading in cases diagnosed as GGG1 prior to robot-assisted radical prostatectomy (RARP).

\section{Patients and methods}

Patients. We retrospectively reviewed 733 patients with $\mathrm{PC}$ who had undergone RARP between October 2010 and May 2018 at our institution. NCB slides prepared elsewhere were routinely reviewed by a urological pathology expert in our hospital. We excluded 130 patients without outside NCB pathology evaluation, 162 patients without second-opinion evaluation, 14 patients without available GGG (outside specimens with inadequate grading, i.e., Gleason $1+2$ ), and 24 patients who received neoadjuvant hormonal therapy. Finally, 403 patients were included in this study. Patient age, body mass index, prostate volume, initial prostate specific antigen (PSA) level, prostate-specific antigen density (PSAD), number of biopsy cores, and positive cores in both initial, and second-opinion diagnoses were reviewed.

Evaluation of Gleason score. The evaluations of NCB by a urological pathology expert in our hospital was performed before RARP. Evaluations of RP specimens were also performed by same urological pathology expert without knowledge of the previous NCB results. In both NCB and RP specimens, GS was assigned to each lesion based on the sum of their primary and secondary tumor patterns, and the highest GS was adapted among the positive lesions. These RP specimens were formalin fixed, sectioned at $4 \mathrm{~mm}$ intervals from apex to base, paraffin embedded, and stained with hematoxylin and eosin. The GS (both outside and second opinion) for each NCB specimen was compared to the GS for corresponding RP specimens, and a GS discrepancy was defined as a difference in GGG category. The five GGG categories were: GGG1, GS 3+3; GGG2, GS 3+4=7; GGG3, GS 4+3=7; GGG4, GS 8; and GGG5, GS 9 or $10(1,8)$.

This retrospective study had formal ethical approval from the Okayama University Institutional Review Board (registration no. 1004) prior to study initiation. All patients provided written informed consent for use of their clinical records.

Statistical analysis. To determine true predictors of upgrading among GGG1 cases, we selected patients who were diagnosed as GGG1 by both the initial and second-opinion pathologists. Patients who could not be evaluated using Prostate Imaging Reporting and Data System version 2 (PI-RADS) score were excluded from this predictor analysis. PI-RADS scores were assigned by a radiologist in our hospital with no knowledge of the pathological results. To evaluate the association between clinical characteristics and upgrade in GGG1 patients, Fisher's exact test was used for categorical variables, and the Mann-Whitney U-test was used for continuous variables. The continuous variables are shown as the median and interquar- tile range (IQR). Predictors of upgrading were analyzed using logistic regression analysis, including age, body mass index, prostate volume, initial PSA, PSAD, PI-RADS score, number of positive cores in both initial and second-opinion diagnoses, and percentage of positive cores in initial and second-opinion diagnoses. Variables with a P-value $<0.05$ in univariate analysis were entered into multivariable analysis. The P-value were two-sided. A P-value $<0.05$ indicated statistical significance for all analyses. All statistical analyses were performed using EZR version 1.36, a graphical user interface for R (12).

\section{Results}

Patient characteristics. Of the 733 cases that underwent RARP at our institution during the study period, 403 patients for which outside opinions, second opinions, and RP reports were available were included in this analysis. Transrectal ultrasound-guided biopsy (TRUS-GB) was performed for diagnosis; no patients underwent magnetic resonance imaging-guided biopsy (MRI-GB) or saturation template biopsy. Clinical characteristics of patients are summarized in Table I. The median GGG was 2 [interquartile range (IQR), 1-4] for first-opinion NCB specimens, whereas the median GGG for second-opinion and RP specimens was 3 (IQR, 2-4). Table II shows the distribution of GGGs among first-opinion, second-opinion, and RP specimens. One case was diagnosed as high-grade prostatic intraepithelial neoplasia (HGPIN) after RARP, which was diagnosed as GGG4 by the initial pathologist and GGG2 by the second pathologist.

Discrepancies in GGG. Discrepancies in GGG between initial and second opinions are shown in Table SI. Of the 403 cases, 147 cases $(36.5 \%)$ had a discrepancy; among these, 89 cases $(60.5 \%)$ were upgraded and 58 cases $(39.5 \%)$ were downgraded after second review. GGG1 diagnoses had the highest rate of agreement $(75 \%)$, while only $50 \%$ agreement was achieved among GGG3 diagnoses between the initial and second opinions. Discrepancies in GGG between first-opinion and RP specimens and between second-opinion and RP specimens are shown in Tables SII and SIII. The agreement rate between GGG was 35.2\% (142 of 403) between first-opinion and RP specimens and $36.5 \%$ (147 of 403) between second-opinion and RP specimens. The first-opinion diagnoses had a larger upgrading rate [41.2\% (166 of 403)] than second-opinion diagnoses did [36.0\% (145 of 403)].

We also investigated GGG concordance between RP specimens and NCB specimens for which a consensus was reached by the first and second pathologists (Table III). The concordance rate was $41.4 \%$ (106 of 256); of the 256 cases, 97 cases $(37.9 \%)$ were upgraded, and 53 cases $(20.7 \%)$ were downgraded upon RP specimen analysis. Notably, $70.9 \%$ (56 of 79) of GGG1 cases were upgraded on RP specimen analysis, 40 (71.4\%) were upgraded to GGG2, 9 (16.1\%) were upgraded to GGG3, 4 (7.1\%) were upgraded to GGG4, and 3 $(5.4 \%)$ were upgraded to GGG5.

Predictors of upgrading. To determine predictors of upgrading among GGG1 diagnoses, we divided GGG1 cases for which the GGG was agreed upon by two pathologists into upgraded $(n=53)$ and non-upgraded $(n=23)$ subgroups. Three cases 
Table I. Demographic and clinical characteristics of the whole cohort.

\begin{tabular}{lc}
\hline Variable & Cases (N=403) \\
\hline Age, median (IQR) & $68(63-72)$ \\
BMI, median (IQR) & $23.7(21.9-33.3)$ \\
Prostate volume, median (IQR) & $27(20-37)$ \\
Initial PSA, median (IQR) & $7.1(5.1-10.4)$ \\
PSAD, median (IQR) & $0.26(0.18-0.43)$ \\
Clinical T stage, n (\%) & \\
T1a & $2(1)$ \\
T1b & $0(0)$ \\
T1c & $87(22)$ \\
T2a & $182(44)$ \\
T2b & $41(10)$ \\
T2c & $68(17)$ \\
T3a & $20(5)$ \\
T3b & $3(1)$ \\
T3c & $0(0)$ \\
Number of biopsy core, median (IQR) & $11(10-14)$ \\
Positive core of 1st opinion, median (IQR) & $3(2-5)$ \\
Positive core of 2nd opinion, median (IQR) & $3(2-5)$ \\
\hline
\end{tabular}

BMI, body mass index; IQR, interquartile range; PSA, prostate specific antigen; PSAD, prostate specific antigen density.

for which the PI-RADS score could not be evaluated were excluded from this analysis. The clinical characteristics of each subgroup are shown in Table SIV. In univariate logistic regression analysis, PSAD and PI-RADS score were identified as statistically significant predictors $(\mathrm{P}=0.009$ and $\mathrm{P}=0.017$, respectively) and were entered into the multivariate logistic regression model. Multivariate analysis revealed that these two variables were independent predictors of upgrading of GGG1 after RARP (PSAD: Odds ratio, 1.10; $\mathrm{P}=0.010$; PI-RADS score: Odds ratio, 1.88; $\mathrm{P}=0.026$ ) (Table IV).

\section{Discussion}

The GGG concordance rate between NCB and RP specimens improved by $6.2 \%$ following a second opinion. However, $71 \%$ of opinion-matched GGG1 cases were upgraded after RARP. Higher PSAD and higher PI-RADS score were independent risk factors for upgrading of GGG1 cases.

GS is the most important parameter for selection of therapeutic management strategies for PC $(1,13)$. Therefore, accuracy of GS determination is critical. Previous studies showed that mandatory second-opinion pathology review by specialized urologic pathologists alters management strategies and improves care in some cases $(2,14,15)$. A major discrepancy between GS assigned by general pathologists and specialized pathologists was observed in $15-41 \%$ of random prostate biopsy specimens $(4,15,16)$. These reports suggested that general pathologists significantly undergraded GS compared with urologic pathologists (16). The adoption of second-opinion review improves the accuracy of GS
Table II. Distribution of GGG among needle-core biopsy specimens.

\begin{tabular}{lccc}
\hline $\begin{array}{l}\text { Gleason } \\
\text { grade }\end{array}$ & $\begin{array}{c}\text { First, } \mathrm{n} \\
(\%)\end{array}$ & $\begin{array}{c}\text { Second, } \\
\mathrm{n}(\%)\end{array}$ & $\begin{array}{c}\text { Pathology, } \\
\mathrm{n}(\%)\end{array}$ \\
\hline Atypical & $0(0)$ & $0(0)$ & $1(0)$ \\
GGG1 $(\leq 3+3)$ & $106(27)$ & $98(24)$ & $33(8)$ \\
GGG2 $(3+4)$ & $102(25)$ & $87(21)$ & $153(37)$ \\
GGG3 $(4+3)$ & $61(15)$ & $61(15)$ & $101(25)$ \\
GGG4 $(8)$ & $90(22)$ & $109(28)$ & $43(11)$ \\
GGG5 $(9,10)$ & $44(11)$ & $48(12)$ & $72(19)$ \\
\hline
\end{tabular}

GGG, Gleason grade group.

between NCB and RP specimens in 5-24\% of cases $(2,17)$. Most of these disagreements involved a single-digit change in GS, and it was estimated that treatment recommendations changed in $9-26 \%$ of these cases (18). In the present study, the concordance between original and second-opinion GGG was similar to that observed in previous studies (63.5\%), and a tendency to upgrade GS after second review was also observed. However, the GS accuracy was improved in only a small number of cases (35.2-36.5\%) after second review compared to previous studies, resulting in relatively lower accuracy upon evaluation of RP specimens (mean, 51.4\%; range, $28-76 \%)(2)$.

The improvement in accuracy was small upon second review due to the fact that the accuracy of Gleason scoring by general pathologists has improved over time. A previous report showed that the concordance between GS assigned by the original pathologist and a second pathologist was significantly higher in the second half of their 13-year study period (16). Although the accuracy of assigning GS is improving in general and among urological pathologists, discrepancies remain a challenge. Grey areas exist between adjacent grades in the Gleason system, particularly between GS 3 and GS 4; thus, interpretation of the border criteria sometimes differs between pathologists. One study revealed that such variation in interpretation of criteria yields a lower rate of agreement: Only $9.9 \%$ of 71 specimens had total agreement among three pathologists, and the rate of total disagreement was $26.8 \%$ (19). Another pathological factor contributing to discrepancy in Gleason scoring is that the International Society of Urological Pathology modified GS revised the definition of Gleason pattern 3 to be very rigorous. Thus, intelligibility of the new standards for the criteria defining Gleason patterns 3 and 4 may influence the upgrading rate (20). In addition, evaluation bias, which is well-documented in the literature, occurs when the GS is based on a single biopsy fragment rather than a consensus of all fragments (21).

In the present study, we selected NCB specimens for which the diagnosis was agreed upon by both the original pathologist and a second pathologist, and investigated the accuracy of these diagnoses compared with those of RP specimens. Although the diagnostic accuracy of opinion-matched cases was $6.2 \%$ higher than that of single-opinion cases, the GGG 
Table III. GGG differences between NCB (1st=2nd) and radical prostatectomy specimen.

\begin{tabular}{|c|c|c|c|c|c|c|}
\hline \multirow{2}{*}{$\begin{array}{l}1 \mathrm{st}=2 \mathrm{nd} \text { GGG } \\
\mathrm{NCB} \text { GGG }\end{array}$} & \multicolumn{5}{|c|}{ Pathology GGG, n (\%) } & \multirow[b]{2}{*}{ Total, $\mathrm{n}$} \\
\hline & GGG1 & GGG2 & GGG3 & GGG4 & GGG5 & \\
\hline GGG1 $(\leq 3+3)$ & $23(29)$ & $40(51)$ & $9(11)$ & $4(5)$ & $3(4)$ & 79 \\
\hline GGG2 (3+4) & $1(2)$ & $40(68)$ & $13(22)$ & $1(2)$ & $4(6)$ & 59 \\
\hline GGG3 (4+3) & $0(0)$ & $12(40)$ & $12(40)$ & $5(17)$ & $1(3)$ & 30 \\
\hline GGG4 (8) & $0(0)$ & $6(10)$ & $23(39)$ & $13(22)$ & $17(29)$ & 59 \\
\hline GGG5 $(9,10)$ & $0(0)$ & $4(14)$ & $5(17)$ & $2(7)$ & $18(62)$ & 29 \\
\hline Total & 24 & 102 & 62 & 25 & 43 & 256 \\
\hline
\end{tabular}

GGG, Gleason grade group; NCB, needle core biopsy.

Table IV. Univariate and multivariate analysis of risk factors for upgrading from Gleason grade group 1.

\begin{tabular}{|c|c|c|c|c|c|c|}
\hline \multirow[b]{2}{*}{ Risk factors } & \multicolumn{2}{|c|}{ Univariate } & \multirow[b]{2}{*}{ P-value } & \multicolumn{2}{|c|}{ Multivariate } & \multirow[b]{2}{*}{ P-value } \\
\hline & Odds ratio & $95 \% \mathrm{CI}$ & & Odds ratio & $95 \% \mathrm{CI}$ & \\
\hline Age (years) & 1.06 & $0.98-1.15$ & 0.126 & & & \\
\hline BMI $\left(\mathrm{kg} / \mathrm{m}^{2}\right)$ & 1.05 & $0.83-1.32$ & 0.692 & & & \\
\hline Prostate volume (ml) & 0.99 & $0.96-1.02$ & 0.641 & & & \\
\hline Initial PSA (ng/ml) & 1.16 & $0.97-1.39$ & 0.113 & & & \\
\hline $\operatorname{PSAD}\left(\mathrm{ng} / \mathrm{ml} / \mathrm{cm}^{3}\right)$ & 1.10 & $1.02-1.18$ & 0.009 & 1.10 & $1.02-1.19$ & 0.010 \\
\hline Clinical T stage (1-3) & 1.29 & $0.51-3.27$ & 0.592 & & & \\
\hline Number of positive cores in 1 st opinion & 0.81 & $0.61-1.06$ & 0.129 & & & \\
\hline Number of positive cores in 2 nd opinion & 0.82 & $0.62-1.09$ & 0.176 & & & \\
\hline$\%$ of positive core in 1 st opinion & 0.99 & $0.96-1.01$ & 0.332 & & & \\
\hline$\%$ of positive core in 2 nd opinion & 0.98 & $0.96-1.01$ & 0.293 & & & \\
\hline PI-RADS score $(1-5)$ & 1.87 & $1.12-3.11$ & 0.017 & 1.88 & $1.08-3.27$ & 0.026 \\
\hline
\end{tabular}

BMI, body mass index; PI-RADS, the prostate imaging reporting and data system; PSA, prostate specific antigen; PSAD, prostate specific antigen density.

of $58.6 \%$ of opinion-matched cases was discordant from that assigned based on RP specimen analysis. Moreover, the second NCB specimen and RP specimen reviews were performed by the same urological pathology expert in our hospital using the same interpretation of criteria. Thus, the inaccuracy might have been caused by sampling error rather than misreading. The multifocal and heterogeneous character of PC makes it difficult to adequately sample the prostate gland (22). A significant statistical sampling variation occurs with the use of a systematic number of biopsy cores in prostate glands that fluctuate in volume; hence, increasing the number of cores improves both PC sampling and accuracy (2). Conversely, the small number of biopsy cores and short length of them might induce overgrading of GS in NCB specimens; 53 (21\%) cases were downgraded after RP despite the opinion-matched pathology.

The use of MRI-GB has been rapidly increasing worldwide as an important tool to improve diagnostic accuracy in PC $(22,23)$. Previous studies reported the GS concordance between MRI-GB and RP specimens to be $57-90 \%$, which is higher than that of TRUS-GB $(22,23)$. MRI-GB also changes the distribution of GGG in men with newly diagnosed PC toward diagnosis of higher-risk disease (24). The cancer detection rate is similar between MRI- and TRUS-GB; however, targeted biopsy allows diagnosis of $30 \%$ more high-risk cancers than systemic biopsy and $17 \%$ fewer low-risk cancers, notably $29 \%$ fewer GGG1 cancers (24). Considering these findings, the present results, which included no MRI-GB cases, are understandable; $71 \%$ of opinion-matched GGG1 cases diagnosed by NCB specimen were upgraded after RARP. Xu et al (23) similarly reported that $74 \%$ (17 of 23) of TRUS-GB GGG1 cases were upgraded following analysis of RP specimens; in contrast, only $20 \%$ (2 of 10) of MRI-GB cases were upgraded. To enhance diagnostic performance, the role of prostate MRI has been increasing (25). Multi-parametric MRI had a specificity of 0.88 and sensitivity of 0.74 for identifying PC, and high PI-RADS scores predicted more than $80 \%$ of cases with significant disease $(26,27)$. The present results suggest that adoption of multi-parametric MRI is more important than mandatory second-opinion pathology review. 
The ability of PSAD to predict biopsy outcome has also been described in previous studies $(27,28)$. Higher PSAD is correlated with higher Gleason score and tumor volume, resulting in shorter progression-free survival following RP (27). A higher PSAD associated with a low GS suggests that TRUS-GB has not hit tumor tissue in many cases. In these cases, template biopsy should be considered for patients with high PSAD. Corcoran et al (29) reported that PSAD was the strongest predictor of GS upgrading between initial biopsy and RP specimen analysis. PSAD was identified as an independent predictor for upgrading in patients with GGG1 based on consensus NCB specimens in the present study, as was PI-RADS score. These two factors therefore are useful not only for predicting biopsy outcomes but also for suggesting clinically significant and aggressive PCs.

The present study had several limitations. First, we included only cases that underwent RARP. Cases that were diagnosed as benign or very low-risk PC were not referred to our institution. Such selection bias may increase the potential for GGG upgrading. Second, we did not have a unified method of biopsy. The number of cores and biopsy location differed by originating institution; furthermore, the length of cancer in positive cores was not analyzed in this study. Third, the urologic pathologist in our hospital performed the Gleason scoring for both NCB review and RP specimens. Therefore, an interpretation bias might have influenced the agreement between initial and subsequent diagnoses.

In conclusion, the concordance rate between $\mathrm{NCB}$ and RP specimen Gleason scoring was improved in opinion-matched cases. However, $71 \%$ of opinion-matched GGG1 cases were upgraded after RARP. Urologists would suggest therapeutic intervention or further biopsy for patients with GGG1 and a high PSAD and/or PI-RADS score.

\section{Acknowledgements}

Not applicable.

\section{Funding}

No funding was received.

\section{Availability of data and materials}

The datasets used and/or analyzed during the current study are available from the corresponding author on reasonable request.

\section{Authors' contributions}

YMa wrote the manuscript, and made substantial contribution to the acquisition of data and data analysis. TS made substantial contributions to the conception and design of the present study, and the interpretation of data. MA, YMi, KW, AGHR and KM made substantial contributions to the analysis and interpretation of data in the present study. YK, MW, HY, TW and YN made substantial contributions to the conception and design of the study, the critical point of discussion and the completion of the manuscript. All authors read and approved the final manuscript.

\section{Ethics approval and consent to participate}

This retrospective study had formal ethical approval from the Okayama University Institutional Review Board (registration no. 1004) prior to study initiation. All patients provided written informed consent for the use of their clinical records. All procedures performed in studies involving human participants were in accordance with the ethical standards of the institutional research committee and with the 1964 Helsinki declaration and its later amendments or comparable ethical standards.

\section{Patient consent for publication}

Not applicable.

\section{Competing interests}

The authors declare that they have no competing interests.

\section{References}

1. Epstein JI, Egevad L, Amin MB, Delahunt B, Srigley JR and Humphrey PA; Grading Committee: The 2014 international society of urological pathology (ISUP) consensus conference on Gleason grading of prostatic carcinoma: Definition of grading patterns and proposal for a new grading system. Am J Surg Pathol 40: 244-252, 2016.

2. Barqawi AB, Turcanu R, Gamito EJ, Lucia SM, O'Donnell CI, Crawford ED, La Rosa DD and La Rosa FG: The value of second-opinion pathology diagnoses on prostate biopsies from patients referred for management of prostate cancer. Int J Clin Exp Pathol 4: 468-475, 2011.

3. Ruijter E, van Leeders G, Miler G, Debruyne F and van de Kaa C: Errors in histological grading by prostatic needle biopsy specimens: Frequency and predisposing factors. J Pathol 192: 229-233, 2000.

4. Brimo F, Schultz L and Epstein JI: The value of mandatory second opinion pathology review of prostate needle biopsy interpretation before radical prostatectomy. J Urol 184: 126-130, 2010.

5. Truesdale MD, Cheetham PJ, Turk AT, Sartori S, Hruby GW, Dinneen EP, Benson MC and Badani KK: Gleason score concordance on biopsy-confirmed prostate cancer: Is pathological re-evaluation necessary prior to radical prostatectomy? BJU Int 107: 749-754, 2011.

6. Weir MM, Jan E and Colgan TJ: Interinstituional pathology consultations. A reassessment. Am J Clin Pathol 120: 405-412, 2003.

7. Epstein JI, Walsh PC and Sanfilippo F: Clinical and cost impact of second-opinion pathology. Review of prostate biopsies prior to radical prostatectomy. Am J Surg Pathol 20: 851-857, 1996.

8. Epstein JI, Zelefsky MJ, Sjoberg DD, Nelson JB, Egevad L, Magi-Galluzzi C, Vickers AJ, Parwani AV, Reuter VE, Fine SW, et al: A contemporary prostate cancer grading system: A Validated alternative to the Gleason score. Eur Urol 69: 428-435, 2016.

9. Loeb S, Folkvaljon Y, Robinson D, Lissbrant IF, Egevad L and Stattin P: Evaluation of the 2015 Gleason grade groups in a nationwide populaton-based cohort. Eur Urol 69: 1135-1141, 2016.

10. Womble PR, Montie JE, Ye Z, Linsell SM, Lane BR and Miller DC; Michigan Urological Surgery Improvement Collaborative: Contemporary use of initial active surveillance among men in Michigan with low-risk prostate cancer. Eur Urol 67: 44-50, 2015.

11. Eggener SE, bandani K, Barocas DA, Barrisford GW, Cheng JS, Chin AI, Corcoran A, Epstein JI, George AK, Gupta GN, et al: Gleason 6 prostate cancer: Translating biology into population health. J Urol 194: 626-634, 2015.

12. Kanda Y: Investigation of the freely available easy-to-use software 'EZR' for medical statistics. Bone Marrow Transplant 48: 452-458, 2013. 
13. Mottet N, Bellmunt J, Bolla M, Briers E, Cumberbatch MG, De Santis M, Fossati N, Gross T, Henry AM, Joniau S, et al: EAU-ESTRO-SLOG Guidelines on Prostate Cancer. Part 1: Screening, diagnosis, and local treatment with curative intent Eur Urol 71: 618-629, 2017.

14. Montironi R, Lopez-Beltran A, Cheng L, Montorsi F and Scarpelli M: Central prostate pathology review: Should it be mandatory? Eur Urol 64: 199-201, 2013.

15. Steinberg DM, Sauvageot J, Piantadosi S and Epstein JI: Correlation of prostate needle biopsy and radical prostatectomy Gleason grade in academic and community settings. Am J Surg Pathol 21: 566-576, 1997.

16. Renshaw AA, Schultz D, Cote K, Loffredo M, Ziemba DE and D'Amico AV: Accurate Gleason grading of prostatic adenocarcinoma in prostate needle biopsies by general pathologists. Arch Pathol Lab Med 127: 1007-1008, 2003.

17. Soga N, Yatabe Y, Kageyama T, Ogura Y and Hayashi N: Review of bioptic scores by central pathologist modifies the risk classification in prostate cancer. Urol Int 95: 452-456, 2015.

18. Townsend NC, Ruth K, Al-Saleem T, Horwitz EM, Sobczak M, Uzzo RG, Viterbo R and Buyyounouski MK: Gleason scoring at a comprehensive cancer center: What's the difference? J Nat Compr Canc Netw 11: 812-819, 2013.

19. McLean M, Srigley J, Banerjee D, Warde P and Hao Y: Interobserver variation in prostate cancer Gleason scoring: Are there implications for the desigh of clinical trials and treatment strategies? Clin Oncol (R Coll Radiol) 9: 222-225, 1997.

20. Usón PLS junior, Macarenco RSES, Oliveira FN and Smaletz O: Impact of pathology review for decision therapy in localized prostate cancer. Clin Med Insights Pathol 10: 1179555717740130, 2017.

21. Klein EA, Cooperberg MR, Magi-Galluzzi C, Simko JP, Falzarano SM, Maddala T, Chan JM, Li J, Cowan JE, Tsiatis AC, et al: A 17-gene assay to predict prostate cancer aggressiveness in the context of Gleason grade heterogeneity, tumor multifocality, and biopsy undersampling. Eur Urol 66: 550-560, 2014.
22. Quentin M, Blondin D, Arsov C, Schimmöller L, Hiester A, Godehardt E, Albers P, Antoch G and Rabenalt R: Prospective evaluation of magnetic resonance imaging guided in-bore prostate biopsy versus systematic transrectal ultrasound guided prostate biopsy in biopsy naïve men with elevated prostate specific antigen. J Urol 192: 1374-1379, 2014.

23. Xu N, Wu YP, Li XD, Lin MY, Zheng QS, Chen SH, Li JF, Wei Y and Xue XY: Risk of upgrading from prostate biopsy to radical prostatectomy pathology: Is magnetic resonance imaging-guided biopsy more accurate? J Cancer 9: 3634-3639, 2018.

24. Siddigui MM, Rais-Bahrami S, Turkbey B, George AK, Rothwax J, Shakir N, Okoro C, Raskolnikov D, Parnes HL, Linehan WM, et al: Comparison of MR/ultrasound fusion-guided biopsy with ultrasound-guided biopsy for the diagnosis of prostate cancer. JAMA 313: 390-397, 2015.

25. Litwin MS and Tan HJ: The diagnosis and treatment of prostate cancer: A review. JAMA 317: 2532-2542, 2017.

26. De Rooij M, Hamoen EH, Futterer JJ, Barentsz JO and Rovers MM: Accuracy of multiparametric MRI for prostate cancer detection: A meta-analysis. AJR Am J Roentgenol 202: 343-351, 2014

27. Washino S, Okochi T, Saito K, Konishi T, Hirai M, Kobayashi Y and Miyagawa T: Combination of prostate imaging reporting and data system (PI-RADS) score and prostate-specific antigen (PSA) density predicts biopsy outcome in prostate biopsy naïve patients. BJU Int 119: 225-233, 2017.

28. Veneziano S, Pavlica P, Compagnone G and Martorana G: Usefulness of the (F/T)/PSA density ratio to detect prostate cancer. Urol Int 74: 13-18, 2005.

29. Corcoran NM, Casey RG, Hong MK, Pedersen J, Connolly S, Peters J, Harewood L, Gleave ME, Costello AJ, Hovens CM and Goldenberg SL: The ability of prostate-specific antigen (PSA) density to predict an upgrade in Gleason score between initial prostate biopsy and prostatectomy diminishes with increasing tumour grade due to reduced PSA secretion per unit tumour volume. BJU Int 110: 36-42, 2012. 